\title{
Terminal Sliding Mode Control of Airship Pitch Channel simplified model
}

\author{
Guoqiang Liang ${ }^{1, \mathrm{a}}$, Yuliang $\mathrm{Chen}^{2}$ and Guangbin $\mathrm{Wu}^{1}$ \\ ${ }^{1}$ Department of control engineering, Naval aeronautical and astronautical University, Yantai, China \\ ${ }^{2}$ Department of Ordnance Science and Technology, Naval aeronautical and astronautical \\ University Yantai, China \\ aliangguoqiang1024@126.com
}

Keywords: airship; pitch channel; adaptive; robustness; stability

\begin{abstract}
To make the response of airship system to be quicker, a kind of terminal sliding mode surface was constructed which can make the pitch angle trace the desired value in a finite time. Also a Lyapunov function was given to prove the sliding mode method is right and reasonable. But detailed simulation result shows that the response is very quick but it has chattering problem which is caused by the loss of integral item in the formation of control law. So a conclusion was got that terminal sliding mode surface should has enough damping ratio in the formation of control law or the system response will has oscillation.
\end{abstract}

\section{Introduction}

Since 1990, airship has widely attract attention from many countries as a low speed cheap aircraft because of its military proportion to be used as a stable platform. And many researchers have turned to the airship flight control research field, not only many traditional control methods ${ }^{[1-6]}$ such as PID control is applied to airship, but also many modern control methods such as robust control, adaptive control and neural network control method is also widely used in airship control system. And the sliding mode variable structure control has been favored by many engineers because of its good robustness and rapidity ${ }^{[7-13]}$. And the terminal sliding mode is attractive because of its limited time convergence characteristics. In this paper, a class of terminal sliding mode surface is introduced to analyze the attitude control problem of the pitching channel of airship model, and the stability of the system is analyzed. Also the simulation is carried out to find out the characteristic of the dynamic response of the airship.

\section{Model Description}

Based on the previous work, the pitch channel model of airship can be described as follows:

$$
M \dot{x}=f(x)+g(x) u
$$

And $x=\left[\begin{array}{llllll}u & w & q & \theta & x & z\end{array}\right], M$ satisfies 


$$
M^{-1}=\left[\begin{array}{llllll}
a_{11} & & a_{13} & & & \\
& a_{22} & & & & \\
a_{31} & & a_{33} & & & \\
& & & 1 & & \\
& & & & 1 & \\
& & & & & 1
\end{array}\right]
$$

The definition of $a_{i j}$ see the definition of $M$ in previous work[3-5].

Choose the expect value of all states $u, w, q, \theta, x, z$ are $u^{d}, w^{d}, q^{d}, \theta^{d}, x^{d}, z^{d}$, define the error variable $e=x-x^{d}, \dot{e}=\dot{x}$, then it hold

$$
M \dot{e}=f(x)+g(x) u
$$

Use the inverse matrix of $M$

$$
\dot{e}=M^{-1} f(x)+M^{-1} g(x) u
$$

To make it convenient for reading, some functions can be written as follows[6-8]

$$
f(x)=\left[\begin{array}{c}
f_{1} \\
f_{2} \\
f_{3} \\
f_{4} \\
f_{5} \\
f_{6}
\end{array}\right], \quad g(x)=\left[\begin{array}{cc}
0 & 1 \\
k_{1} & 0 \\
k_{2} & 0 \\
0 & 0 \\
0 & 0 \\
0 & 0
\end{array}\right], u=\left[\begin{array}{ll}
u_{1} & u_{2}
\end{array}\right]^{T}
$$

where

$$
\left[\begin{array}{l}
f_{1} \\
f_{2} \\
f_{3} \\
f_{4} \\
f_{5} \\
f_{6}
\end{array}\right]=\left[\begin{array}{c}
-\left(m+m_{33}\right) w q+Q\left[C_{X 1} \cos ^{2} \alpha+C_{X 2} \sin (2 \alpha) \sin (\alpha / 2)\right. \\
\left(m+m_{11}\right) q u+m a_{z} q^{2}+Q\left[C_{z 1} \cos (\alpha / 2) \sin (2 \alpha)+C_{z 2} \sin (2 \alpha)+C_{z 3} \sin (\alpha) \sin (|\alpha|)\right] \\
-m a_{z} w q(-r v)+Q\left[C_{M 1} \cos (\alpha / 2) \sin (2 \alpha)+C_{M 2} \sin (2 \alpha)+C_{M 3} \sin (\alpha) \sin (|\alpha|)\right]-a_{z} \sin \theta W \\
q \\
u \cos \theta+w \sin \theta \\
-u \sin \theta+w \cos \theta
\end{array}\right]
$$

\section{Define[9]}

$$
M^{-1} f(x)=\left[\begin{array}{c}
f_{a 1} \\
f_{a 2} \\
f_{a 3} \\
f_{a 4} \\
f_{a 5} \\
f_{a 6}
\end{array}\right]=\left[\begin{array}{c}
a_{11} f_{1}+a_{13} f_{3} \\
a_{22} f_{2} \\
a_{31} f_{1}+a_{33} f_{3} \\
f_{4} \\
f_{5} \\
f_{6}
\end{array}\right]
$$

And 


$$
g(x) u=\left[\begin{array}{c}
u_{2} \\
k_{1} u_{1} \\
k_{2} u_{1} \\
0 \\
0 \\
0
\end{array}\right]
$$

Then the system can be written as follows

$$
\left[\begin{array}{c}
\dot{u} \\
\dot{w} \\
\dot{q} \\
\dot{\theta} \\
\dot{x} \\
\dot{z}
\end{array}\right]=\left[\begin{array}{c}
f_{a 1} \\
f_{a 2} \\
f_{a 3} \\
f_{a 4} \\
f_{a 5} \\
f_{a 6}
\end{array}\right]+\left[\begin{array}{c}
a_{11} u_{2}+a_{13} k_{2} u_{1} \\
a_{22} k_{1} u_{1} \\
a_{31} u_{2}+a_{33} k_{2} u_{1} \\
0 \\
0 \\
0
\end{array}\right]
$$

\section{Robust Sliding Mode Control of Attitude}

Assume that the airship moves at constant speed and attitude, and assume angle of pitch $\theta^{d}=2 / 57.3$, then define sliding mode surface[10-11]:

$$
s_{1}=3 c_{1}\left(\theta-\theta^{d}\right)^{1 / 3}+q
$$

Differentiate the sliding mode surface

$$
\dot{s}_{1}=c_{1} q\left(\theta-\theta^{d}\right)^{-2 / 3}+\dot{q}=c_{1}\left(\theta-\theta^{d}\right)^{-2 / 3} q+a_{31} f_{1}+a_{33} f_{3}+a_{31} u_{2}+a_{33} k_{2} u_{1}
$$

Consider decoupling control, $u_{1}$ controls vertical movement and $u_{2}$ controls forward movement, then design:

$$
u_{2}=\text { Cons }
$$

Assume $a_{31} f_{1}+a_{33} f_{3}$ is bounded and the boundary is known, then[12-15]:

$$
a_{31} f_{1}+a_{33} f_{3}<d_{1} a_{33} k_{2}
$$

If the boundary is unknown, choose the value of the boundary $\hat{d}_{1}$, the design control law as follow:

$$
u_{1}=u_{1 b}=-k_{0} s_{1}-\hat{k}_{1} s_{1}-\hat{k}_{2} q\left(\theta-\theta^{d}\right)^{-2 / 3}-\hat{d}_{1} \operatorname{sign}\left(s_{1}\right)-\hat{k}_{4} u_{2}
$$

then

$$
\begin{aligned}
s_{1} \dot{s}_{1} \leq & -a_{1} s_{1} s_{1}+\left(c_{1}-a_{33} k_{2} \hat{k}_{2}\right) q s_{1}\left(\theta-\theta^{d}\right)^{-2 / 3}+\left(a_{31} f_{1}+a_{33} f_{3}-a_{33} k_{2} \hat{d}_{1}\right) s_{1} \\
& +\left(a_{31}-a_{33} k_{2} \hat{k}_{4}\right) u_{2} s_{1}+\left(a_{1}-a_{33} k_{2} k_{0}-a_{33} k_{2} \hat{k}_{1}\right) s_{1} s_{1}
\end{aligned}
$$

It can be got by simplifying 


$$
\begin{aligned}
s_{1} \dot{s}_{1} \leq & -a_{1} s_{1} s_{1}+\left(c_{1}-a_{33} k_{2} \hat{k}_{2}\right) q s_{1}\left(\theta-\theta^{d}\right)^{-2 / 3}+d_{1}\left|s_{1}\right|-a_{33} k_{2} \hat{d}_{1}\left|s_{1}\right| \\
& +\left(a_{31}-a_{33} k_{2} \hat{k}_{4}\right) u_{2} s_{1}+\left(a_{1}-a_{33} k_{2} k_{0}-a_{33} k_{2} \hat{k}_{1}\right) s_{1} s_{1}
\end{aligned}
$$

Define:

$$
\tilde{d}_{1}=d_{1}-a_{33} k_{2} \hat{d}_{1}
$$

then

$$
\dot{\tilde{d}}_{1}=-a_{33} k_{2} \dot{\hat{d}}_{1}
$$

Where

$$
\begin{aligned}
& \dot{\hat{d}}_{1}=\Gamma_{d} s_{1} \\
& \quad \dot{\hat{k}}_{2}=\Gamma_{2} s_{1} q\left(\theta-\theta^{d}\right)^{-2 / 3}
\end{aligned}
$$

So

$$
\left[\frac{1}{2 \Gamma_{d} a_{33} k_{2}}(\tilde{d})^{2}\right]^{\prime}=-\tilde{d} s_{1}
$$

choose a Lyapunov function as[16-19]:

$$
V_{c}=\frac{1}{2} s_{1}^{2}+\sum_{i=1, i \neq 3}^{4}\left[\frac{1}{2 \Gamma_{i} a_{33} k_{2}}\left(\tilde{k_{i}}\right)^{2}\right]+\frac{1}{2 \Gamma_{i} a_{33} k_{2}}(\tilde{d})^{2}
$$

So

$$
\dot{V}_{c} \leq-a_{1} s_{1}^{2} \leq 0
$$

It is not difficult to prove that system is stable in hypothetical condition.

\section{Numerical Simulation}

The system is proved to be stable by theoretical derivation as above, in order to test the stability of the system, this section uses SIMULINK tool case in MATLAB to the simulation.

In this section, choose $u_{2}=10000$, assume that the initial height is 1 meter, assume that attitude angle is 10 degree, and choose $d_{1}=0.01$, simulation results are as follows:

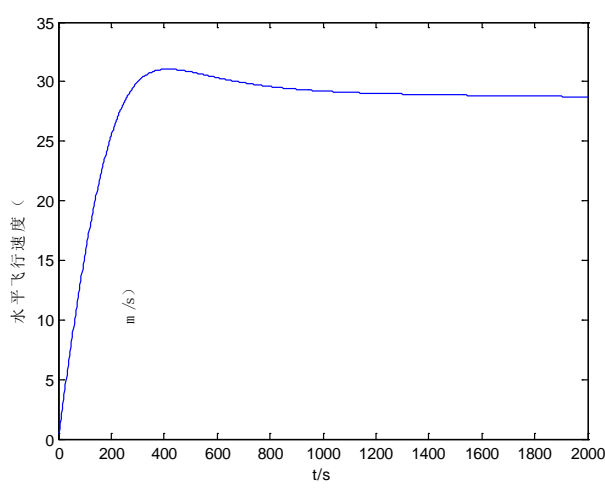

Fig. 1 Forward Velocity

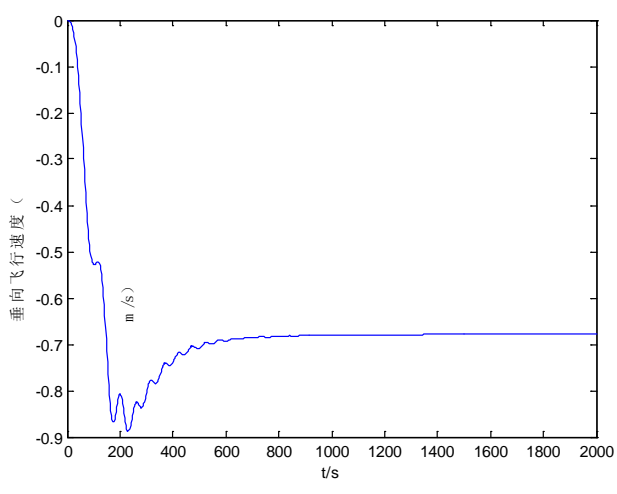

Fig. 2 Vertical Velocity 


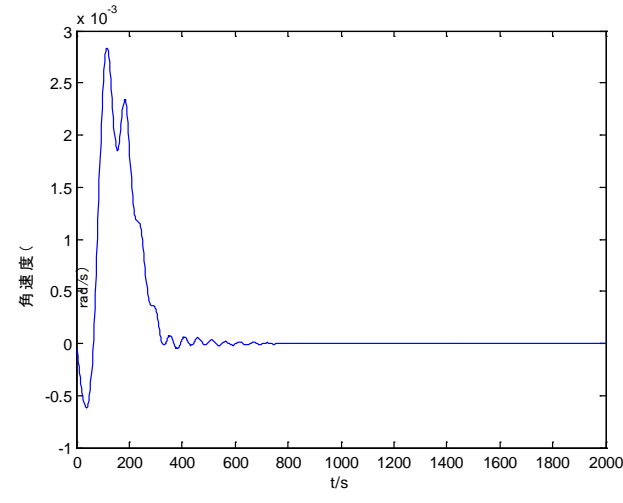

Fig. 3 Angle Velocity

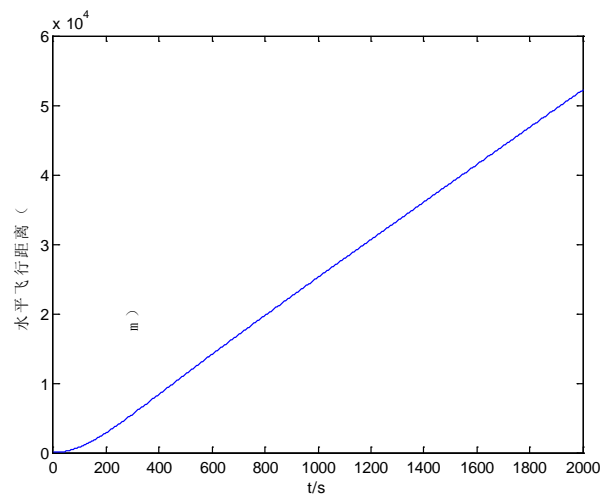

Fig. 5 Flying Distance

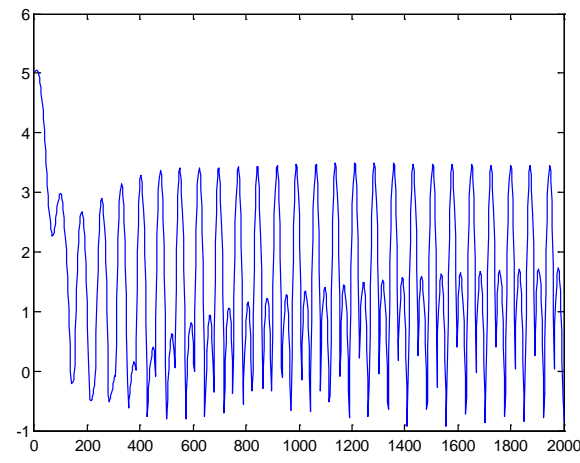

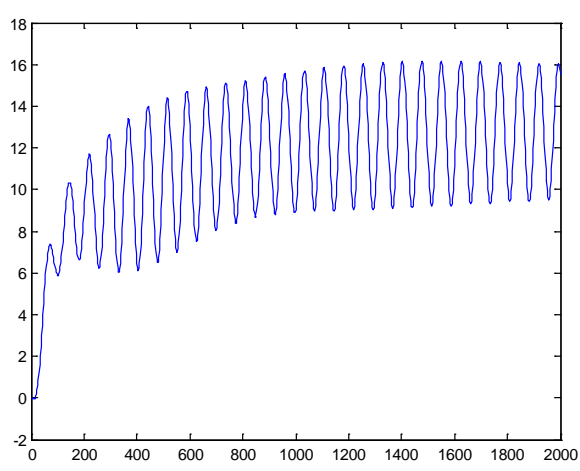

Fig. 4 Pitch Angle

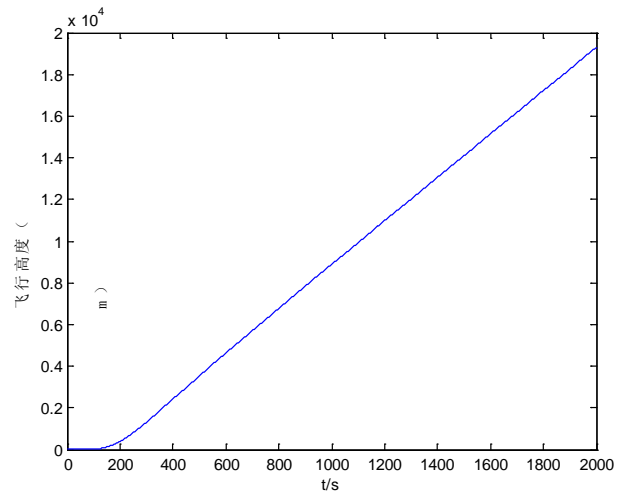

Fig. 6 Height

Fig. 7 Actuator Angle

According to above simulation result, we can find that the pitch angle can trace desired value 10 deg in 100s, but there exists big oscillations. And also it will cause the actuator angle has chattering problem. It means that the terminal method should integrated with integral method, which can reduce steady state error.

\section{Conclusion}

A novel kind of terminal sliding mode surface was proposed to solve the problem that the tracking of pitch angle of airship is too slow. And the whole system with proposed method was proved to be stable, but simulation result shows that the dynamic performance is not ideal. The reason is that although the response speed is improved, but there is another problem appeared that the system response has oscillation which is caused by the lacking of damping ratio in the formation 
of whole control law. So how to increase the damping ration and without change the terminal sliding mode surface greatly is the main point of our future work.

\section{References}

[1] Elfes A., S.Bergerman, M.Ramos. A semi-autonomous robotic airship for environmental monitoring missions. IEEE International Conference on Robotics and Automation. 1998. Leuven, Belgium.

[2] Gomes S B V, Romos J J G. Airship Dynamic Modeling for Autonomous Operation. Proceedings of the 1998 IEEE International Conference on Robotics and Automation. 1998, 3462-3467.

[3] L.B.Tuckerman.Inertia Factors of Ellipsoids for Use in Airship Design. Naca Reports.2006,14(3):45 50

[4] E.C.de Paiva,S.S.Bueno,Influence of Wind Speed on Airship Dynamics, Journal of Guidance,Control and Dynamics.2002,25(6):116 124

[5] Sergio B.Varella Gomes and Josue Jr.G.Ramos.Airship dynamic modeling for autonomous operation.Proceedings of the 2003 IEEE. International Conference on Robotics\&Automation.2003:5 14

[6] J.S.Uhlman,N.E.Fine,D.C.Kring.Calculation of the Added Mass and Damping Forces on Supercavitating Bodies.The 4th International Symposium on Cavitation,California,2001:7 13

[7] D.Clarke.Calculation of the Added Mass of Elliptical Cylinders in Shallow Water.Ocean Engineering.2001,28(4):61 72

[8] C.J.Atkinson,R.G.Urso.Modeling of Apparent Mass Effects for the Real-Time Simulation of a Hybird Airship.AIAA Modeling and Simulation Technologies Conference and Exhibit,Keystone.2006:21 32

[9] Yokomaku Y.The Stratospheric Platform Airship R\&D Program of Japan. The 2nd Stratospheric Platform Systems Workshop,Tokyo Japan.2000:7 13

[10] S.P.Jones,J.D.Laurier.Aerodynamic Estimation Techniques for Aerostats and Airships.AIAA Lighter-than-Air Systems Conference,Annapolis,2004: 88 94

[11] M.T.Soylemeza,N.Munrob,H.Bakic.Fast Calculation of Stabilizing PID Controllers.Automatic,2003,39(7):121 126

[12] Etkin B, Theory of the flight of Airplanes in Isotropic Turbulence Review an Extension,AGARD Rept.1961:372

[13] David K. Schmidt,James Stevens,Jason Roney.Dynamic Modeling,Control, and Station-Keeping Guidance of A Large High-Altitude“Near-Space” Airship.AIAA Guidance,Navigation,and Control Conference and Exhibit. 2006-6781 